\title{
Changes in Plasma FcRIII Demonstrate Increasing Receptor Production during Late Pregnancy and after Preterm Birth
}

\author{
ROBERT CARR, TOM W. J. HUIZINGA, MARION KLEIJER, AND JOHN M. DAVIES \\ The University Department of Haematology, Royal Liverpool Hospital, Liverpool, United Kingdom [R.C., \\ J.M.D.J, and Central Laboratory of the Netherlands Red Cross Blood Transfusion Service and Laboratory for \\ Experimental and Clinical Immunology, University of Amsterdam, Amsterdam, The Netherlands \\ [T.W.J.H., M.K.]
}

\begin{abstract}
We have previously described reduced expression of the $\mathrm{Fc} \gamma$ receptor type III on the cell membrane (M-FcRIII) of neutrophils from very preterm neonates. To investigate the mechanism underlying this reduced receptor expression, we have measured neutrophilderived soluble FcRIII (S-FcRIII) in the plasma of fetuses and neonates from 19 wk gestation. S-FcRIII in fetal plasma and in preterm neonates born before 32 wk gestation was consistently low [mean 13.6 $\pm 1.2 \%$ (mean adult $\mathrm{S}-\mathrm{FcRIII}=\mathbf{1 0 0} \%$, range $\mathbf{3 0}-\mathbf{2 4 0} \%$ )]. In utero, $\mathrm{S}-\mathrm{FcRIII}$ starts to rise from 33 wk and increases more than 4-fold to reach adult levels by term. S-FcRIII measured sequentially in preterm infants born as early as 24 wk of gestation showed a rapid postnatal increase to reach adult levels within 3 wk of birth. The changes in S-FcRIII paralleled changes in M-FcRIII expression on the cell surface. These observations point to a reduced rate of FcRIII production by fetal neutrophils as opposed to an increase in receptor release. The parallel increase in S-FcRIII and M-FcRIII suggests that there may be a programmed activation of FcRIII synthesis within individual cells late in the 3rd trimester of fetal development. In addition, FcRIII production may be switched on early by preterm birth. (Pediatr Res 32: 505-508, 1992)
\end{abstract}

\section{Abbreviations}

FcRIII, Fc $\gamma$ receptor type III

M-FcRIII, neutrophil membrane-associated $\mathrm{Fc} \gamma$ receptor type III

S-FcRIII, soluble plasma $\mathrm{Fc} \gamma$ receptor type III

RDS, respiratory distress syndrome of prematurity

$\mathrm{CI}$, confidence interval

Little is known about the processes underlying functional maturation of neutrophil immunity during ontogeny. In fullterm neonates, most components of the immune system are still immature: Ig production is limited in quantity and is class restricted $(1,2)$, and neutrophils are defective in their ability to migrate and kill microorganisms (3). There are few studies of neutrophil functional ability in very immature preterm neonates born before $30 \mathrm{wk}$ of gestation. Those published have demonstrated impaired phagocytosis (4), defective killing (5), and re-

Received November 7, 1991; accepted June 25, 1992

Correspondence: Dr. Robert Carr, Division of Haematology, United Medical and Dental School, St. Thomas' Hospital, Lambeth Palace Road, London SE1 7EH, United Kingdom

Supported by a grant from the Mersey Regional Health Authority. duced peak chemiluminescence responses $(6,7)$ compared with well, full-term neonates and adult controls. There have been no studies comparing the postnatal changes that occur in infants born prematurely at less than $30 \mathrm{wk}$ of gestation with the normal neutrophil functional development that occurs in utero during the last trimester of pregnancy. Consequently, there is little known about the control of normal neutrophil ontogeny and the changes brought about by preterm birth.

We have recently found that the neutrophils from preterm neonates born before 32 wk express reduced numbers of FcRIII, in contrast to normal FcRIII expression by neutrophils from term neonates (8). Neutrophil FcRIII is encoded by the gene FcRIII-1 (9), which is only expressed in neutrophils. It is linked to the neutrophil cell membrane by a phosphatidylinositol anchor and is shed from the plasma membrane after cell activation (10). A soluble form of FcRIII of neutrophil origin (S-FcRIII) is present in high concentration in the plasma (11). Patients lacking the FcRIII-1 gene express no FCRIII on the neutrophil cell membrane (M-FcRIII) and have no S-FcRIII in their plasma but have normal expression of FcRIII on their natural killer cells and macrophages (encoded by the gene FcRIII-2) $(12,13)$. Neutrophil membrane FcRIII is involved in the binding of IgGopsonized particles and is able to trigger the lytic machinery of the neutrophil $(14,15)$.

To investigate the mechanism underlying reduced M-FcRIII expression by neutrophils from preterm neonates and to chart how this aspect of neutrophil immunity evolves during fetal development, we measured S-FcRIII in neonates and fetuses between $19 \mathrm{wk}$ of gestation and term. In addition, we examined the influence of premature birth by monitoring S-FcRIII and MFcRIII during the early postnatal development of infants born between 24 and $30 \mathrm{wk}$ of gestation.

\section{MATERIALS AND METHODS}

Study population. Neutrophils and plasma were collected from 31 preterm neonates soon after birth and at intervals during the first weeks of life. The median gestational age was 29 wk (range 23-36 wk). Initial samples were collected within $36 \mathrm{~h}$ of birth from infants who had not been transfused with red cells, platelets, or fresh frozen plasma; follow-up samples were collected more than 48 h after any transfusion. Neonates were defined as "well" if they had no evidence of infection and did not require mechanical ventilation for pulmonary insufficiency. Neonates with RDS had typical $x$-ray changes of hyaline membrane disease and required intensive ventilatory support. Neutrophils and plasma were collected from 15 healthy term neonates at the time of birth by elective cesarean section $(n=12)$ or spontaneous vaginal delivery $(n=3)$. All deliveries by cesarean section were performed under epidural anesthesia before the onset of labor. Fetal plasma 
samples were collected from 13 unstressed and untransfused fetuses, median gestational age 22 wk (range 19-33 wk), during routine diagnostic percutaneous umbilical cord blood sampling.

The study was approved by and neonatal blood sampling was in accordance with the Liverpool Maternity Hospital Ethical Committee guidelines.

Preparation of samples. Blood from preterm neonates was obtained from umbilical artery catheters or peripheral veins and from the umbilical cord vessels of term neonates and anticoagulated with $10 \mathrm{U} / \mathrm{mL}$ heparin. Plasma was separated and frozen within $1 \mathrm{~h}$ of sampling. Neutrophils were separated on a sodium metrizoate/dextran gradient (Polyprep, Nycomed Ltd., Buckingham, UK). Residual red cells were removed by hypotonic lysis and the neutrophils resuspended in RPMI medium with $\mathrm{Ca}^{++}$ and $\mathrm{Mg}^{++}$(Flow Laboratories, Hertfordshire, UK). The cell suspensions and all reagents were kept on ice. Viability, by trypan blue exclusion, was $>95 \%$ after separation.

$M-F c R I I I$ expression. M-FcRIII expression was quantitated by indirect immunofluorescence using MAb Leu-11b (Becton Dickinson, Abingdon, Oxford, UK) and FITC-conjugated goat anti-mouse IgG (Becton Dickinson). Surface stained cells were fixed in $1 \%$ paraformaldehyde and analyzed on a Becton Dickinson FACS Analyzer I. Each sample was processed in parallel with cells from a healthy adult control. The mean fluorescence intensity distribution of 5000 neutrophils was measured on a 3decade logarithmic scale and expressed as a fluorescence index (mean fluorescence neonate neutrophils/mean fluorescence adult control neutrophils, \%).

$S$-FCRIII concentration. S-FcRIII was determined by a sandwich RIA as described by Huizinga et al. (11). CLBFcRgran 1 (an anti-FcR MAb) was covalently coupled to cyanogen bromideactivated Sepharose 4B beads (10 mg protein to $1 \mathrm{~g}$ Sepharose) and diluted 1:600 in PBS containing EDTA $(10 \mathrm{mM})$ and Tween $(0.1 \% \mathrm{wt} / \mathrm{vol})$. Five hundred $\mu \mathrm{L}$ of this Sepharose suspension were incubated with 10,20 , and $40 \mu \mathrm{L}$ of plasma from a test neonate/fetus or with serial doubling dilutions (320 to $1.25 \mu \mathrm{L})$ of a plasma pool prepared from 38 normal donors for $18 \mathrm{~h}$ at room temperature by head-over-head rotation in 2 -mL polystyrene tubes. Each test was performed in triplicate. The Sepharose beads were washed five times with $1.5 \mathrm{~mL}$ saline. The anti-FcR MAb BW209/2 was radiolabeled according to the Iodogen method, following the manufacturer's instructions (Pierce, Rockford, IL). Ten $\mu \mathrm{g}$ of labeled MAb were diluted with $500 \mathrm{~mL}$ PBS containing EDTA $(10 \mathrm{mM})$, Tween-20 (0.1\% wt/vol) and $1 \%$ normal goat serum to prevent nonspecific binding. Five hundred $\mu \mathrm{L}$ of the labeled MAb solution were added to the tubes and incubated for $5 \mathrm{~h}$ with rotation. The tubes were washed five times and radiation measured in a multi-gamma counter (LKB Instruments Inc., Gaithersburg, MD). The amount of S-FcRIII was determined by comparison with the standard calibration curve, generated using the serial dilutions of the pooled plasma as described (11). The range of S-FcRIII in the plasma of 25 randomly chosen patients admitted to a general hospital was $40-$ $180 \%$. The range of S-FcRIII in the plasma of the 38 healthy volunteer donors was 30-240\%. Assuming that one BW209/2 $\mathrm{MAb}$ binds to one FcRIII molecule, $100 \%$ would represent a plasma concentration of $5 \mathrm{nM}$.

Statistical analysis. Study groups are described by mean and SEM. Differences between groups are described by $95 \% \mathrm{CI}$, and statistical significance was determined using two-tailed $t$ test.

\section{RESULTS}

$S$-FCRIII and gestational age (Figs. 1 and 2). Plasma concentrations of S-FCRIII were measured in 13 unstressed fetuses, 31 preterm neonates within $36 \mathrm{~h}$ of birth, and 15 term neonates at birth. Between 19 and 32 wk of gestation, plasma S-FcRIII was at a consistently low level, below the normal adult range of $30-$ $240 \%$. Infants born from $33 \mathrm{wk}$ onward had progressively increased S-FcRIII at birth, with the majority of term neonates

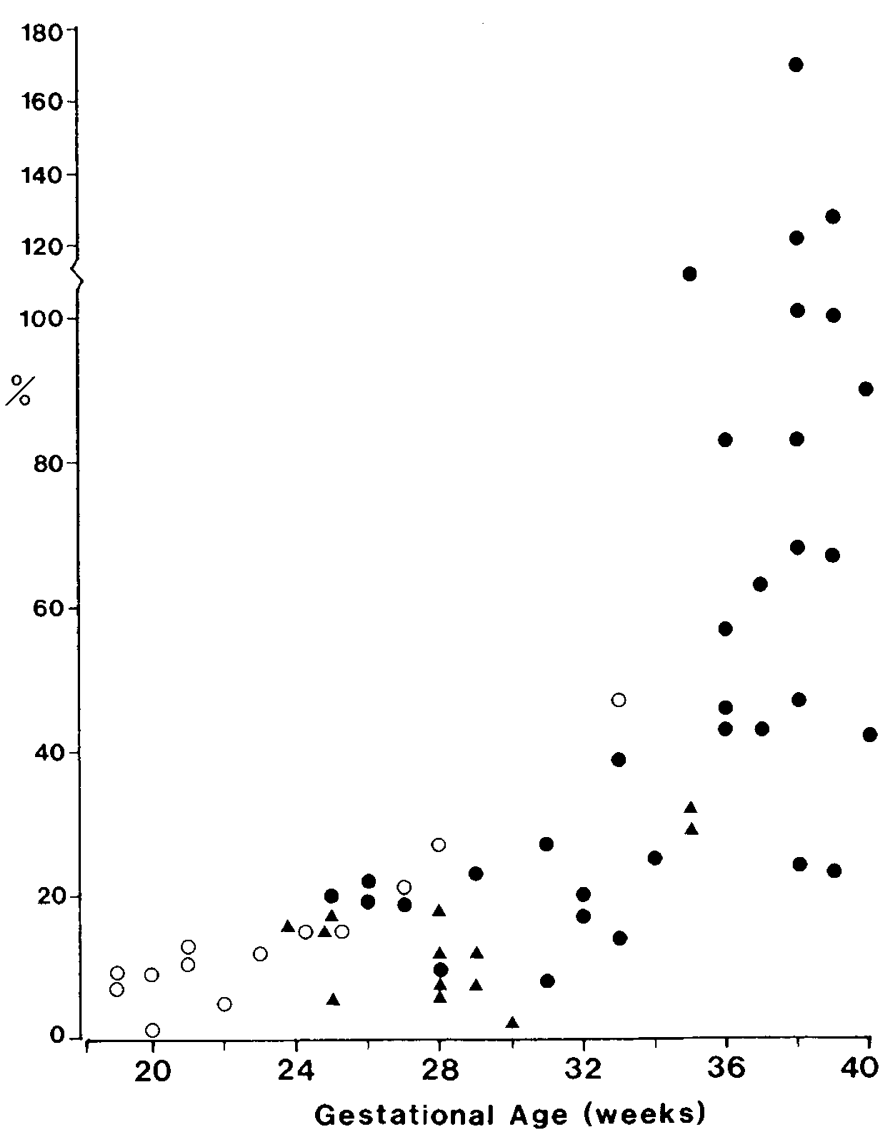

Fig. 1. S-FcRIII in utero or at birth, by gestational age. $O$, Fetal samples; - , well neonates; $\boldsymbol{\Delta}$, neonates with severe RDS. Each point represents an individual subject. Ordinate, concentration of S-FcRIII (\%) compared to a standard plasma pool from 38 normal donors. (The SFCRIII concentration in the standard plasma pool is taken to be $100 \%$.)

having normal adult levels. Levels in fetuses measured after 24 wk of gestation were comparable to neonates of similar gestational age, showing that there was no sudden alteration in $S$ FcRIII at the time of birth.

Fetal S-FcRIII between 19 and 23 wk gestation tended to be lower than neonatal and fetal S-FcRIII between 24 and 32 wk of gestation $(8.3 \pm 1.4 \%$ versus $15.3 \pm 1.3 \%$; mean difference $7.0 \%$, $95 \% \mathrm{Cl} 2.1-11.9 \% ; p<0.02)$. Preterm neonates of $<32 \mathrm{wk}$ gestation with severe RDS tended to have lower S-FcRIII levels than preterm neonates who were clinically well $(10.9 \pm 1.6 \%$ versus $18.8 \pm 1.9 \%$; mean difference $7.9 \%$, CI $2.4-13.5 \% ; p<$ 0.01 ). The reason for infants with RDS having lower plasma concentrations of S-FcRIII is not clear, but may represent greater immaturity of neutrophil immunity in this group.

M-FCRIII and gestational age (Fig. 2). We have previously reported that M-FCRIII expression is reduced in neonates born at $<32$ wk of gestation compared with term neonates and adults (8). When M-FcRIII and S-FcRIII were measured simultaneously in neonates in the present study, gestational age-related changes in M-FcRIII expression on the neutrophil cell membrane paralleled S-FcRIII in the plasma. There was no significant change in M-FcRIII between 24 and 32 wk. However, between 33 and 36 wk in utero, M-FcRIII expression increased to reach adult levels by term.

There was quite marked variation in M-FcRIII expression between individual infants. We have previously shown, and confirmed in the present study, that expression of M-FcRIII is transiently down-modulated by sepsis. S-FcRIII appears not to be acutely altered by clinical stress and therefore may be a better index of overall neutrophil maturity.

Postnatal changes in S-FCRIII and M-FcRIII (Fig. 3). Preterm 


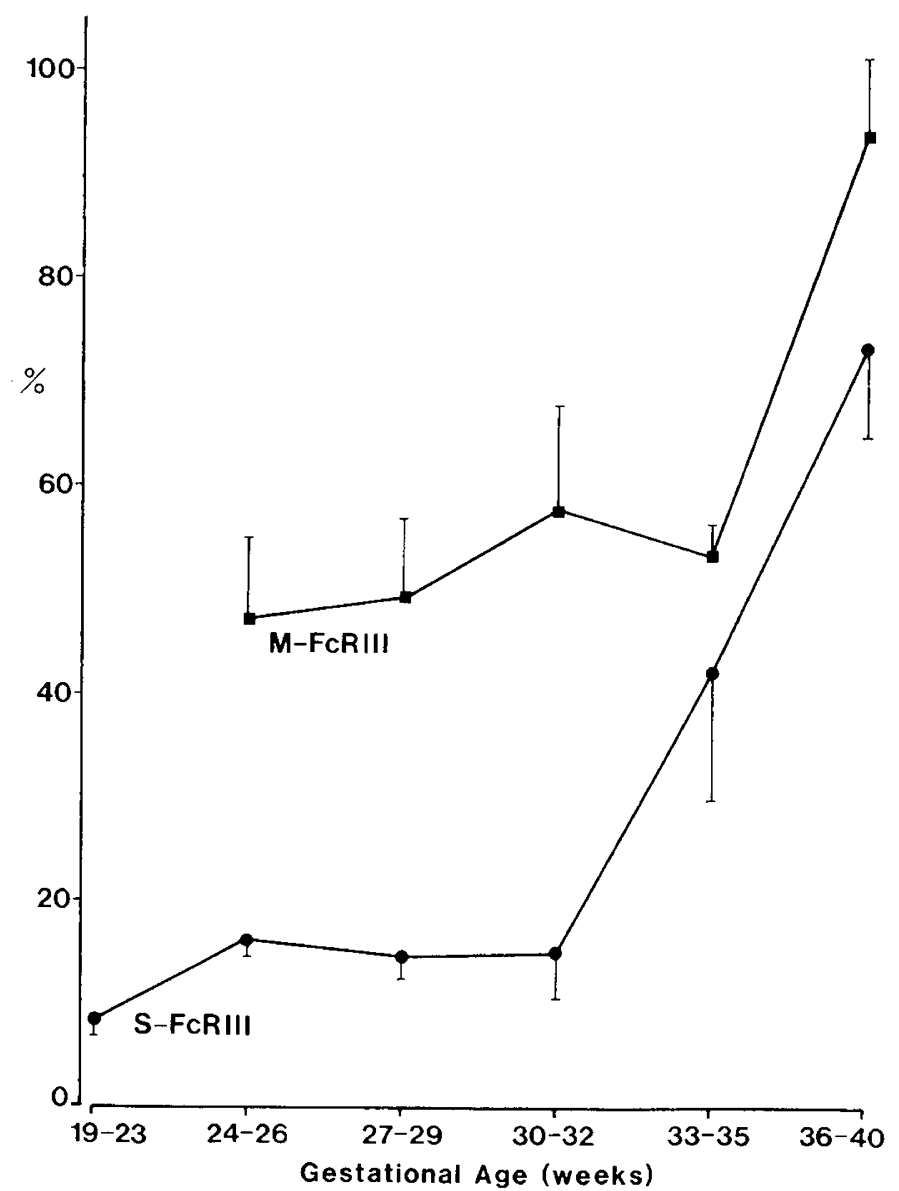

Fig. 2. S-FcRIII and M-FcRIII in utero and at birth, by gestational age. Mean values \pm SEM (bars). Ordinate, $\%$ of mean adult value. There was no significant change in mean neonate S-FcRIII or M-FcRIII measured between 24 and $32 \mathrm{wk}$ of gestation. Fetal neutrophils were not available for M-FcRIII measurement.

infants born between 24 and $30 \mathrm{wk}$ of gestation were monitored during their early postnatal development. After birth, there was a rapid and parallel increase in S-FcRIII in the plasma and MFcRIII on the cell surface. Both S-FcRIII and M-FcRIII were significantly increased by the 5 th-7th postnatal day (mean increase in S-FcRIII 34.1\%, CI 23.4-44.7\%; $p<0.001$; mean increase in M-FcRIII $33.0 \%$, CI $13.9-52.2 \%$; $p<0.01$ ). The majority of infants had reached adult levels within 3 wk after birth (i.e. equivalent to gestational ages of $27-33 \mathrm{wk}$ ).

The postnatal rate of increase in S-FcRIII was not determined by gestational age or clinical status. In contrast, M-FcRIII expression did tend to remain depressed in a few infants with recurrent infections or persistent lung disease.

\section{DISCUSSION}

We have previously described reduced expression of M-FcRIII on the neutrophils of preterm neonates (8). It was unclear from that observation whether diminished M-FcRIII represented reduced receptor production or increased release from the cell surface as a consequence of in vivo cell activation.

In the present study, we found that the plasma concentration of S-FcRIII is at a consistently low level between 19 and 32 wk of gestation. During a normal pregnancy, S-FcRIII increases abruptly from $33 \mathrm{wk}$ to reach normal adult levels by term. In infants born prematurely, even as early as $24 \mathrm{wk}$ of gestation, there is a rapid postnatal increase in S-FcRIII to reach adult levels within 3 wk of birth. The observed changes in S-FcRIII parallel changes in M-FcRIII expression on the cell surface.

The new observation that low levels of M-FcRIII on the cell

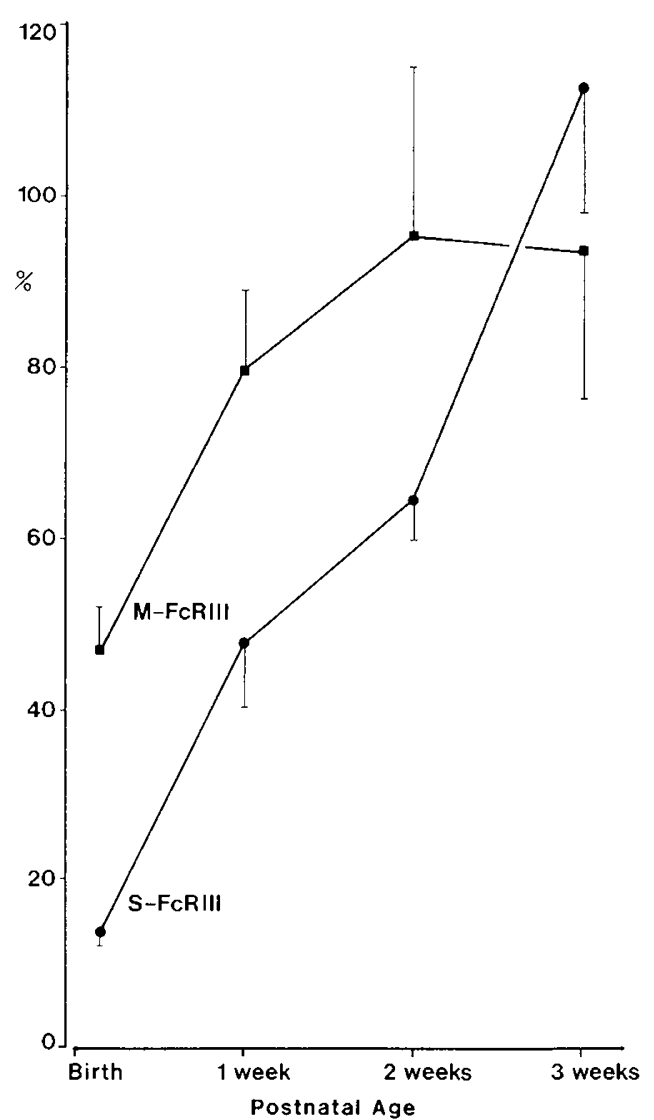

Fig. 3. Changes in S-FcRIII and M-FcRIII during the first 3 postnatal wk in preterm neonates born between 24 and $30 \mathrm{wk}$ of gestation. Mean values $\pm \operatorname{SEM}$ (bars). Ordinate, $\%$ of mean adult value.

surface are associated with very low levels of S-FcRIII in the plasma would favor a reduced rate of receptor production within individual cells as the underlying mechanism. Active synthesis of FcRIII is known to occur in normal mature neutrophils (16, 17 ), and continuing protein synthesis is required to maintain normal FcRIII numbers on the cell membrane (18). In congenital FcRIII-1 deficiency and in paroxysmal nocturnal hemoglobinuria, conditions in which functional FcRIII synthesis is absent or defective, there are absent or low levels of FcRIII both on the neutrophil cell membrane and in the plasma $(10,13,19)$. The alternative explanation, increased receptor shedding, would seem less likely to result in the low plasma levels of S-FcRIII observed if the underlying rate of receptor synthesis was normal.

We speculate that the parallel increase in S-FcRIII and MFcRIII, which occurs between 33 and 36 wk of gestation, may indicate a programmed activation of receptor synthesis within individual cells. An external trigger for this maturation step may be provided by the cytokines granulocyte colony stimulating factor or granulocyte/macrophage colony stimulating factor. Both increase the expression of FcRIII on neutrophils in vitro by a mechanism that appears to require new receptor synthesis (20). Moreover, high concentrations of both granulocyte colony stimulating factor and granulocyte/macrophage colony stimulating factor have been reported in cord blood from term infants (21).

Further investigation of this phenomenon, by direct measurement of neutrophil FcRIII receptor synthesis and mRNA transcription during fetal development, may reveal control mechanisms amenable to therapeutic intervention for the future enhancement of neonate neutrophil function.

Acknowledgments. The authors thank Professor R. W. I. Cooke and Dr. A. M. Weindling for permission to study neonates under their care at the Liverpool Maternity Hospital. We thank Profes- 
sor C. H. Rodeck at the Institute of Obstetrics and Gynaecology, Royal Postgraduate Medical School, London, for providing fetal plasma samples. Thanks also go to Diane Pumford for technical assistance.

\section{REFERENCES}

1. Andersson U, Bird AG, Britton S, Palacios R 1981 Humoural and cellular immunity in humans studied at the cell level from birth to two years of age. Immunol Rev 57:5-38

2. Nagaoki T, Miyawaki T, Ciobaru R, Yachie A, Uwadana N, Moriya N, Taniguchi N 1981 Maturation of B cell differentiation ability and T cell regulatory function during child growth assessed in a nocardia water soluble mitogen-driven system. J Immunol 126:2015-2019

3. Hill HR 1987 Biochemical, structural and functional abnormalities of polymorphonuclear leukocytes in the neonate. Pediatr Res 22:375-382

4. Al-Hadithy H, Addison IE, Goldstone AH, Cawley JC, Shaw JC 1981 Defective neutrophil function in low-birth-weight, premature infants. J Clin Pathol 34 $366-370$

5. Gahr M, Blanke R, Speer CP 1985 Polymorphonuclear leukocyte function in term and preterm newborn infants. Biol Neonate 48:15-20

6. Peden DB, VanDyke K, Ardekani A, Mullett MD, Myerberg DZ, VanDyke C 1987 Diminished chemiluminescent responses of polymorphonuclear leukocytes in severely and moderately preterm neonates. J Pediatr 111: 904-906

7. Driscoll MS, Thomas VL, Ramamurthy RS, Castro DT 1990 Longitudinal evaluation of polymorphonuclear leukocyte chemiluminescence in premature infants. J Pediatr 116:429-434

8. Carr R, Davies JM 1990 Abnormal FcRIII expression by neutrophils from very preterm neonates. Blood 76:607-611

9. Ravetch JV, Perussia B 1989 Alternative membrane forms of Fc $\gamma$ RIII (CDI6) on human natural killer cells and neutrophils. J Exp Med 170:481-497

10. Huizinga TWJ, van der Schoot CE, Jost C, Klaassen R, Kleijer M, von dem Borne AEGKr, Roos D, Tetteroo PAT 1988 The PI-linked receptor FcRIII is released on stimulation of neutrophils. Nature 333:667-669

11. Huizinga TWJ, de Haas $M$, Kleijer $M$, Nuijens $J H$, Roos D, von dem Borne
AEGKr 1990 Soluble Fc $\gamma$ receptor III in human plasma originates from release by neutrophils. J Clin Invest 86:416-423

12. Clark MR, Liu L, Clarkson SB, Ory PA, Goldstein IM 1990 An abnormality of the gene that encodes neutrophil Fc receptor III in a patient with systemic lupus erythematosus. J Clin Invest 86:341-346

13. Huizinga TWJ, Kuijpers RWAM, Kleijer M, Schulpen TWJ, Cuypers TAM, Roos D, von dem Borne AEGKr 1990 Maternal genomic neutrophil FcRIII deficiency leading to neonatal isoimmune neutropenia. Blood 76: $1927-1932$

14. Huizinga TWJ, van Kemenade F, Koenderman L, Dolman KM, von dem Borne AEGKr, Tetteroo PAT, Roos D 1989 The 40-kDa Fc $\gamma$ receptor (FcRII) on human neutrophils is essential for the IgG-induced respiratory burst and IgG-induced phagocytosis. J Immunol 142:2365-2369

15. Huizinga TWJ, Dolman KM, van der Linden NJM, Kleijer M, Nuijens JH, von dem Borne AEGKr, Roos D 1990 Phosphatidylinositol-linked FcRIII mediates exocytosis of neutrophil granule proteins, but does not mediate initiation of the respiratory burst. J Immunol 144:1432-1437

16. Jack RM, Fearon DT 1988 Selective synthesis of mRNA and proteins by human peripheral blood neutrophils. J Immunol 140:4286-4293

17. Jost CR, Huizinga TWJ, de Goede R, Fransen JAM, Tetteroo PAT, Daha MR, Ginsel LA 1990 Intracellular localization and de novo synthesis of FcRIII in human neutrophil granulocytes. Blood 75:144-151

18. Edwards SW, Watson F, MacLeod R, Davies J 1990 Receptor expression and oxidase activity in human neutrophils: Regulation by granulocyte-macrophage colony-stimulating factor and dependence upon protein biosynthesis. Biosci Rep 10:393-401

19. Jost CR, Gaillard ML, Fransen JAM, Daha MR, Ginsel LA 1991 Intracellular localization of glycosyl-phosphatidylinositol-anchored CD67 and FcRIII (CDI6) in affected neutrophil granulocytes of patients with paroxysmal nocturnal hemoglobinuria. Blood 78:3030-3036

20. Buckle A-M, Hogg N 1989 The effect of IFN- $\gamma$ and colony-stimulating factors on the expression of neutrophil cell membrane receptors. J Immunol 143 : 2295-2301

21. Laver J, Duncan E, Abboud M, Gasparetto C, Sahdev I, Warren D, Bussel J, Auld P, O'Reilly RJ, Moore MAS 1990 High levels of granulocyte and granulocyte-macrophage colony-stimulating factors in cord blood of normal full-term neonates. J Pediatr 116:627-632 